\title{
VECTOR SOLITONS IN MODE LOCKED FIBRE LASERS
}

\author{
S. V. Sergeyev, S. K. Turitsyn
}

Aston Institute of Photonic Technologies, Aston University,

Birmingham, B4 7ET, UK

E-mail: s.sergeyev@aston.ac.uk

Mode-locked lasers emitting a train of femtosecond pulses called dissipative solitons are an enabling technology for metrology, high-resolution spectroscopy, fibre optic communications, nano-optics and many other fields of science and applications. Recently, the vector nature of dissipative solitons has been exploited to demonstrate mode locked lasing with both locked and rapidly evolving states of polarisation. Here, for an Erbium doped fibre laser mode locked with carbon nanotubes, we demonstrate the first experimental and theoretical evidence of a new class of vector solitons (fundamental, multipulsing, bound states etc.) characterised by different polarisation attractors including double-scroll chaotic polarisation attractor substantially different from Lorenz, Rössler and Ikeda strange attractors [1-5]. The underlying physics comprises a long time scale coherent coupling of two polarization modes. The observed phenomena, apart from the fundamental interest, provide a base for advances in secure communications, trapping and manipulation of atoms and nanoparticles, control of magnetization in data storage devices and many other areas.

1. Sergeyev S. V., Mou Ch., Turitsyna E. G. et al. // Light: Science \& Applications. (in press) (2013)

2. Tsatourian V., Sergeyev S. V., Mou Ch. et al. // Scientific Reports. (in press) (2013)

3. Mou Ch., Sergeyev S. V., Rozhin A., Turitsyn S. K. // Optics Express, in press (2013)

4. Sergeyev S. V., Mou Ch., Rozhin A., Turitsyn S. K. // Optics Express. 2012. 20, P. 27434-27440.

5. Mou Ch., Sergeyev S. V., Rozhin A., Turistyn S. // Optics Lett. 2011. 36, P. 3831-3833. 